\title{
Novel evaporation experiment to determine soil hydraulic properties
}

\author{
K. Schneider ${ }^{1}$, O. Ippisch ${ }^{2, *}$, and K. Roth $^{1}$ \\ ${ }^{1}$ Institute of Environmental Physics, University of Heidelberg, Heidelberg, Germany \\ ${ }^{2}$ Institute of Parallel and Distributed Systems, University of Stuttgart, Stuttgart, Germany \\ * formerly: Interdisciplinary Center for Scientific Computing, University of Heidelberg, Germany
}

Received: 24 April 2006 - Published in Hydrol. Earth Syst. Sci. Discuss.: 29 June 2006

Revised: 21 September 2006 - Accepted: 29 October 2006 - Published: 9 November 2006

\begin{abstract}
A novel experimental approach to determine soil hydraulic material properties for the dry and very dry range is presented. Evaporation from the surface of a soil column is controlled by a constant flux of preconditioned air and the resulting vapour flux is measured by infrared absorption spectroscopy. The data are inverted under the assumptions that (i) the simultaneous movement of water in the liquid and vapour is represented by Richards' equation with an effective hydraulic conductivity and that (ii) the coupling between the soil and the well-mixed atmosphere can be modelled by a boundary layer with a constant transfer resistance. The optimised model fits the data exceptionally well. Remaining deviations during the initial phase of an experiment are thought to be well-understood and are attributed to the onset of the heat flow through the column which compensates the latent heat of evaporation.
\end{abstract}

\section{Introduction}

Movement of soil water is usually described by Richards' equation (Jury et al., 1991). A crucial part of this are the hydraulic material properties, in particular the soil water characteristic $\theta_{\ell}\left(\psi_{m}\right)$ and the hydraulic conductivity function $K\left(\theta_{\ell}\right)$. These properties are difficult to measure directly (Topp and Miller, 1966) which led to the development of inverse methods. Most popular today is multi-step outflow (Eching et al., 1994; van Dam et al., 1994) which evolved from one-step outflow (Parker et al., 1985). Although we do not focus on the outflow method per se and its well-known practical problems and limitations, we briefly discuss the fundamental issues demanding the usage of a new method. In outflow methods, gas pressure $p_{g}$ in the soil sample equals ambient atmospheric pressure, as is the case in soils, while

Correspondence to: K. Schneider

(klaus.schneider@iup.uni-heidelberg.de) the pressure $p_{\ell}$ in the liquid phase at the lower end of the sample is reduced in one or more steps. Correspondingly, the matric potential $\psi_{m}=p_{\ell}-p_{g}$ in the sample is reduced and the resulting flow of water is recorded. Obviously, these methods are fundamentally limited to $\psi_{m}>-100 \mathrm{kPa}$ since $p_{\ell}$ must be larger than the vapour pressure of water. Practical limitations like the permeability of the phase separator at the lower boundary are more strict and typically lead to $\psi_{m}>-20 \mathrm{kPa}$. The method is thus only applicable for the range of moderately negative potentials, hence to the rather wet range of soils. This is no real limitation for many processes which primarily operate in this range, including groundwater recharge and solute transport through soils to groundwater. Other processes, however, cannot be described reliably with material properties estimated from such a limited potential range. The reason for this is that the corresponding parameterisations are empirical relations with no physical foundation. Hence, extrapolation is not possible without strong assumptions on the porous media. Notorious examples where representations for the dry range are required include plant water uptake, soilatmosphere coupling, and optimal dry-land farming.

A seemingly simple way to circumvent the fundamental limitation of multi-step outflow measurements would be to keep $p_{\ell}$ constant at ambient atmospheric pressure and to increase $p_{g}$. Since there is no fundamental limit to increasing $p_{g}, \psi_{m}$ can be made arbitrarily negative. However, the water phase now is in a state completely different from that in a natural soil with the same value of $\psi_{m}$. This is easily appreciated by considering a tensiometer in the two situations. Since the relation between the water contents of these two states is unknown, the parameters are not transferable.

The limitations of the classical multi-step outflow experiments for estimating hydraulic material properties can be overcome by evaporation experiments, as they allow virtually unlimited values of the matric potential (by making the air above the surface dry). More precisely, evaporation experiments are a natural complement to MSO: They are sensitive

Published by Copernicus GmbH on behalf of the European Geosciences Union. 


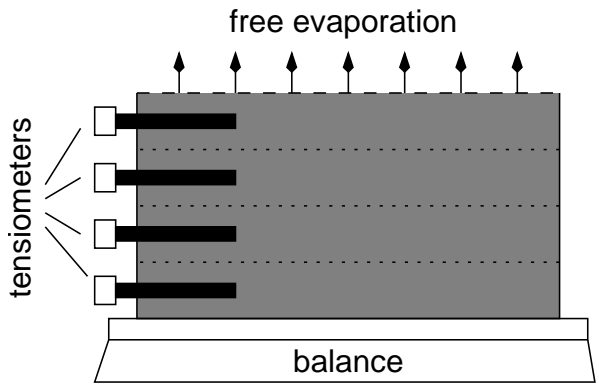

Fig. 1. Schematic of the standard setup of evaporation experiments.

for strongly negative potentials but their accuracy deteriorates rapidly as potentials approach 0 , the range where MSO works best. Gardner and Miklich (1962) were the first to propose such experiments. Their setup was basically retained in later studies (Wendroth et al., 1993; Tamari et al., 1993; Romano and Santini, 1999). A typical experimental setup is shown in Fig. 1. The soil is initially saturated, and then free evaporation is allowed to start. At times $t_{i}$, the potentials $\psi_{m_{i j}}$ at heights $z_{j}$ are measured with tensiometers and the cumulative outflow is monitored using a balance. Additionally, at the end of the experiment the total residual water is measured gravimetrically, thus obtaining the real water content $\theta_{i}$ for each measurement time $t_{i}$. There arise a number of fundamental difficulties with this approach, however: (i) The most severe issue are the tensiometers required for measuring the matric potential. This again sets a lower limit for the applicable matric potential. While the exact value of this limit depends on the location of the uppermost tensiometer as well as on the soil hydraulic properties, it is typically on the order of $-100 \mathrm{kPa}$ for water tensiometers. If the potential falls below the air-entry value of the tensiometer or below the vapour pressure of water, whichever is higher, then water is released from the tensiometer into the soil. This leads to a disturbance of the measurements that may be quite dramatic. (ii) A severe technical challenge results from the very small potential gradients in regions where the hydraulic conductivity is still high. Here, the accuracy of the tensiometers becomes limiting. As a consequence, the method becomes inaccurate near saturation. (iii) Finally, weighing for determining the water flux becomes increasingly difficult as the flux decreases. Dangling cables and air movement, e.g. due to air conditioning, or dust become significant sources of uncertainty and demand special precautions.

To overcome these drawbacks, we retain the basic idea of an evaporation experiment but take a new experimental approach that gets rid of the balance and of the tensiometers. The basic idea is to force evaporation with a well-controlled potential in the head space above the soil column and to accurately measure the resulting vapour flow. The data are then inverted for the coefficients of some parameterisation of the soil hydraulic properties with an accurate model that also ac- counts for the coupling between soil and head space. For the material properties, we chose the Mualem-Brooks-Corey parameterisation, which was shown in Ippisch et al. (2006) to be well suited for fine-textured soils. We assumed a surface boundary layer with a constant transfer resistance.

In this study we focus on technical issues of the new method, intending to give a proof-of-concept. A detailed analysis of the inversion method to further investigate the parameter estimation procedure will be provided in a later study.

\section{Theory}

We model the soil column as a uniform one-dimensional medium and assume that its soil water characteristic may be described by the Brooks-Corey parameterisation

$\Theta_{\ell}\left(\psi_{m}\right)=\frac{\theta_{\ell}\left(\psi_{m}\right)-\theta_{r}}{\theta_{s}-\theta_{r}}= \begin{cases}{\left[\psi_{m} / \psi_{e}\right]^{-\lambda}} & ; \psi_{m}<\psi_{e}, \\ 1 & ; \psi_{m} \geq \psi_{e}\end{cases}$

and its hydraulic conductivity function by the corresponding Mualem parameterisation

$K\left(\Theta_{\ell}\right)=K_{s} \Theta_{\ell}^{\tau+2+2 / \lambda}$.

To model vapour transport through the soil column, we assume local thermodynamic equilibrium. Then, the molar water vapour content $v_{g}^{w}$ is given by (Rawlins and Campbell, 1986)

$v_{g}^{w}=\frac{p_{s}^{w}(T)}{R T} \exp \left(\frac{\psi_{m} V_{m}^{w}}{R T}\right)$,

where $V_{m}^{w}$ is the molar volume of liquid water, and $p_{s}^{w}(T)$ the partial pressure of water vapour over pure liquid water at temperature $T$. It can be described with Magnus' formula (Murray, 1967) as

$p_{s}^{w}(T)=610.78 \mathrm{~Pa} \exp \left(\frac{17.2694(T-273.16 \mathrm{~K})}{T-35.86 \mathrm{~K}}\right)$.

These relations can also be used to calculate the equivalent matric potential from a given water vapour concentration.

The equivalent flux $\boldsymbol{j}_{g}^{w}$ of liquid water transported by diffusion of water vapour is given by

$\boldsymbol{j}_{g}^{w}=-V_{m}^{w} D_{g}^{w} \nabla v_{g}^{w}$,

which we can reformulate, using the chain rule, as

$\boldsymbol{j}_{g}^{w}=-V_{m}^{w} D_{g}^{w}\left(\frac{\partial v_{g}^{w}}{\partial T} \nabla T+\frac{\partial v_{g}^{w}}{\partial \psi_{m}} \nabla \psi_{m}\right)$.

If we neglect the temperature dependent part, approximate the vapour by an ideal gas, and use Eq. (3) we get:

$\boldsymbol{j}_{g}^{w}=-D_{g}^{w} \frac{p_{s}^{w}(T) V_{m}^{w^{2}} \exp \left(\frac{\psi_{m} V_{m}^{w}}{R T}\right)}{[R T]^{2}} \nabla \psi_{m}$. 
Next, we describe the reduction $\xi$ of the effective diffusion coefficient in the porous medium by the simple model (Jin and Jury, 1996)

$\xi\left(\theta_{\ell}\right)=\frac{\theta_{\ell}^{2}}{\theta_{s}^{2 / 3}}$.

The diffusion of water vapour is not hindered as much by liquid films as that of other gases, because water can condensate on one side and re-evaporate on the other (Philip and de Vries, 1957). Therefore the saturated water content $\theta_{s}$ was used for $\theta_{\ell}$ in the tortuosity model yielding the relation $D_{g}^{w}=\theta_{s}^{4 / 3} D_{g, \text { atm }}^{w}$, where $D_{g \text {, atm }}^{w}$ is the diffusion coefficient for water vapour in free air. This assumption may lead to an overestimation of water vapour transport in the soil. However, as liquid water transport is dominant while the soil is wet the effect should be minor. We finally obtain

$\boldsymbol{j}_{g}^{w}=-D_{g, \text { atm }}^{w} \frac{\theta_{s}^{4 / 3} p_{s}^{w}(T) V_{m}^{w^{2}} \exp \left(\frac{\psi_{m} V_{m}^{w}}{R T}\right)}{[R T]^{2}} \nabla \psi_{m}$

which has the same form as the Buckingham-Darcy flux

$\boldsymbol{j}_{\ell}^{w}=-K_{\ell}\left(\theta_{\ell}\right) \nabla \psi_{m}$.

Hence, Richards' equation may be enhanced to include vapour transport in the soil column by writing

$$
\begin{aligned}
\frac{\partial \theta_{\ell}}{\partial t} & =\nabla \cdot\left[K_{g}\left(\psi_{m}\right) \nabla \psi_{m}+K_{\ell}\left(\theta_{\ell}\right) \nabla\left[\psi_{m}-\rho_{\ell}^{w} g z\right]\right] \\
& \approx \nabla \cdot\left[\left[K_{g}\left(\psi_{m}\right)+K_{\ell}\left(\theta_{\ell}\right)\right] \nabla\left[\psi_{m}-\rho_{\ell}^{w} g z\right]\right]
\end{aligned}
$$

with

$$
K_{g}\left(\psi_{m}\right)=D_{g, \text { atm }}^{w} \frac{\theta_{s}^{4 / 3} p_{s}^{w}(T) V_{m}^{w^{2}} \exp \left(\frac{\psi_{m} V_{m}^{w}}{R T}\right)}{[R T]^{2}} .
$$

The approximation in Eq. 11 can be made because the vapour term will only significantly contribute at very low matric potentials where the gravity term is negligible.

A crucial step is the representation of the upper boundary. We model it as a diffusive layer of constant thickness $r_{b}$ and assume that the time scale of diffusion across this layer is much smaller than the time scale on which the boundary condition changes. This appears reasonable since the time scale of diffusion, given by $r_{b}^{2} /\left[2 D_{g, \text { atm }}^{w}\right]$, is some $0.1 \mathrm{~s}$ for a layer thickness of $2 \mathrm{~mm}$. The vapour flux across such a layer is given by

$$
j_{\text {boundary }}^{w}=-\frac{V_{m}^{w} D_{g, \text { atm }}^{w}}{R T} \frac{p_{\exp }^{w}-p_{s}^{w}(T) \exp \left(\frac{\psi_{m}^{b} V_{m}^{w}}{R T}\right)}{r_{b}}
$$

where $p_{\exp }^{w}$ is the partial pressure of water vapour in the wellmixed head space above the soil column and $\psi_{m}^{b}$ the matrix potential at the soil surface. We comment that, by definition, the processes in this layer are not resolved well. In particular its physical location is not defined, i.e., the fraction of the

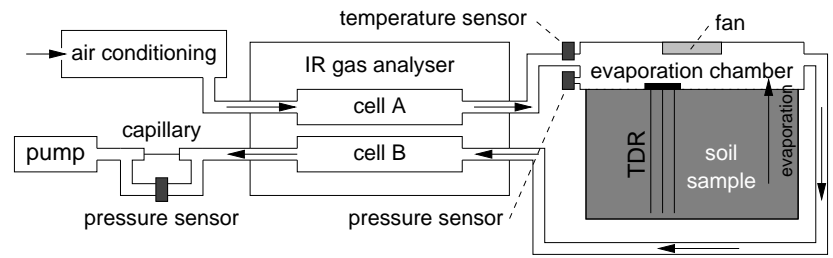

Fig. 2. Experimental setup. Evaporation takes place into a gastight head space (evaporation chamber) above the soil surface. Air is flowing through it to take away the water. Water vapour molar fraction is measured before and after the head space. The air is conditioned before it enters the chamber to set a well defined boundary condition. The gas flow is measured with a capillary and controlled with a vacuum pump with adjustable speed.

layer that is within the soil column, and the porosity of the respective parts of the soil. However, for the type of thin layer we consider here all these complicating factors only enter as a constant of proportionality. Hence, we make $r_{b}$ a fitting parameter that absorbs all these factors. Obviously, its value then cannot be interpreted physically anymore.

\section{Experimental setup}

The soil sample is contained in a PVC cylinder of $81 \mathrm{~mm}$ radius and $100 \mathrm{~mm}$ height. The bottom of the column is closed. The top of the soil column is closed by a $30 \mathrm{~mm}$ high gas-tight head space (evaporation chamber, Fig. 2). A constant flow of air is established through the head space to remove the water vapour and thereby set the potential. Filters prevent dirt from entering the measurement system. The difference of water vapour content before and after the evaporation chamber and the air flow through the chamber quantify the water flux at the upper boundary of the soil sample, while the relative humidity and the temperature in the evaporation chamber define the equivalent matric potential of the air. An infrared absorption gas analyser simultaneously measures the water vapour molar fraction $x$ (mole/mole) before and after the soil sample. Temperature $T$ is measured at the air inlet of the chamber, the total pressure $p$ in the chamber. Inside the chamber the air is mixed with a fan to ensure uniform water vapour content and thus a well-defined potential. The gas flow $q$ is measured by the pressure jump across a capillary with known conductivity. It is controlled by a vacuum pump with adjustable speed. A time domain reflectometry (TDR) sensor (Robinson et al., 2003) is installed vertically in the soil column to measure the total water content. The individual parts of the setup are described in the following.

\subsection{Air conditioning}

To enable a well-defined boundary condition, the air is conditioned before it enters the evaporation chamber, i.e. its water 

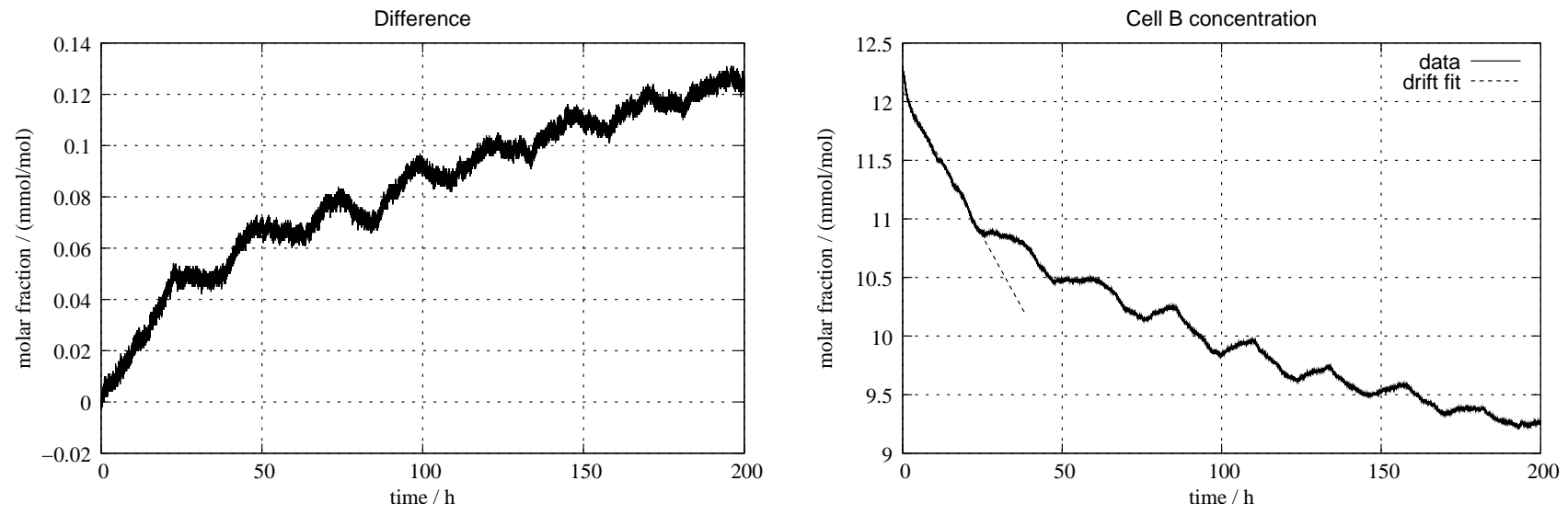

Fig. 3. Water vapour molar fraction measurements of the gas analyser with conditioned air at $10^{\circ} \mathrm{C}$ dew point flowing simultaneously through both cells of the instrument. The difference (left) is very stable while the absolute molar fraction (right) is drifting faster, with about $-49 \mu \mathrm{mol} \mathrm{mol}^{-1} \mathrm{~h}^{-1}$ on the first day (dashed line).

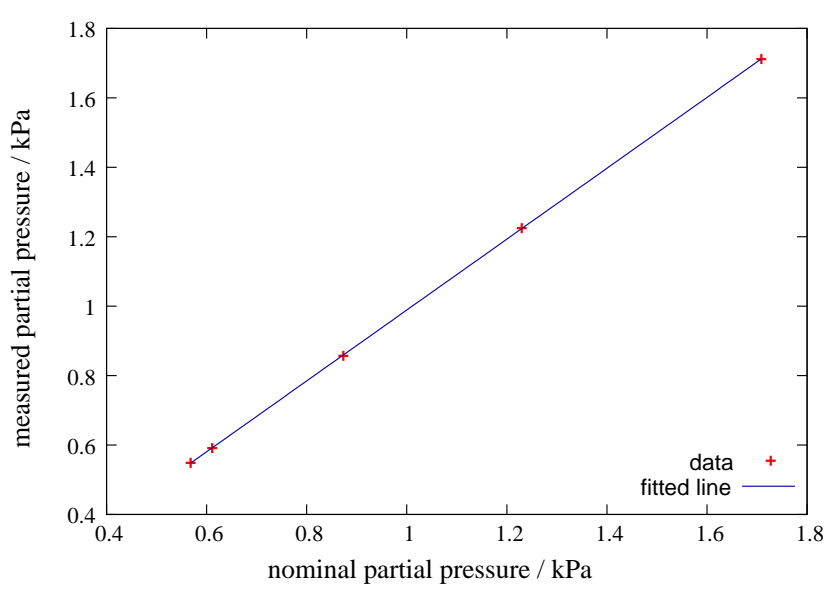

Fig. 4. Linearity and calibration check of the gas analyser. Null gas was flowing through cell $\mathrm{A}$ and air with defined water vapour molar fraction through cell $\mathrm{B}$. The nominal cell $\mathrm{B}$ molar fraction was varied and the measured partial pressure $p_{\text {meas }}$ recorded.

vapour content is set to a defined value. This is done with a cold trap. The air flows through an aluminium box with flow channels. The box is cooled by Peltier elements. Abundant water freezes out in the box, thus the outgoing air has a defined water vapour content. The current through the Peltier elements is regulated based on the temperature of the gas at the outlet of the box by a controller. After the box the air is passively warmed to ambient temperature.

As time passes, more and more water freezes out at the walls. Therefore, there are two identical coolers. If one box becomes frozen up, the incoming air stream is switched to the other box by magnetic valves, and the first box is defrosted, so it is ready again to use when the second one is frozen up.

\subsection{Infrared gas analyser}

The Li-Cor LI-7000 infrared gas analyser used for measuring water vapour content uses the $2595 \mathrm{~nm}$ absorption band. The instrument has a thermal light source and uses an optical bandpass filter to select the appropriate wavelength. No spectral information is needed. Two cells A and B independently measure water vapour molar fraction.

Calibration measurements are required for zero point, span and zero absorption value. The calibration procedure is to let known gases flow through the cells of the instrument, wait for the measurements to stabilise and then execute the corresponding user calibration functions.

Calibration gas is generated with a dew point generator: Air is bubbled through a water bath whose temperature is precisely controlled by a series of peltier thermoelectric coolers. The accuracy of water vapour content of the generated air stream is $\pm 0.2^{\circ} \mathrm{C}$ between $0^{\circ} \mathrm{C}$ and $50^{\circ} \mathrm{C}$, drift is specified as less than $0.02^{\circ} \mathrm{C}$. Additionally, water free null gas (here, $\mathrm{N}_{2} 4.6, \mathrm{H}_{2} \mathrm{O}<5 \mathrm{ppm}$ (volume)) is used for zero point calibration.

To test the accuracy of the gas analyser, the dew point generator was set to $10^{\circ} \mathrm{C}$ and the conditioned air was then flown through both cells, resulting in a zero molar fraction difference. Results are shown in Fig. 3. Noise is very low (about $0.01 \mathrm{mmol} / \mathrm{mol}$ in cell B measurement and $0.005 \mathrm{mmol} / \mathrm{mol}$ in molar fraction difference). In accordance with the statement of the manufacturer, the molar fraction difference is much more stable than the absolute value. Cell B drift is about $0.05(\mathrm{mmol} / \mathrm{mol}) / \mathrm{h}$, molar fraction difference drift about $0.002(\mathrm{mmol} / \mathrm{mol}) / \mathrm{h}$. Thus the instrument is very precise. Drift can be kept low by regular calibration.

To check the calibration and the linearity of the instrument, water-free null gas was used as reference gas flowing through cell $\mathrm{A}$ and calibration gas with a defined dew point, generated with the dew point generator, was flowing through cell 


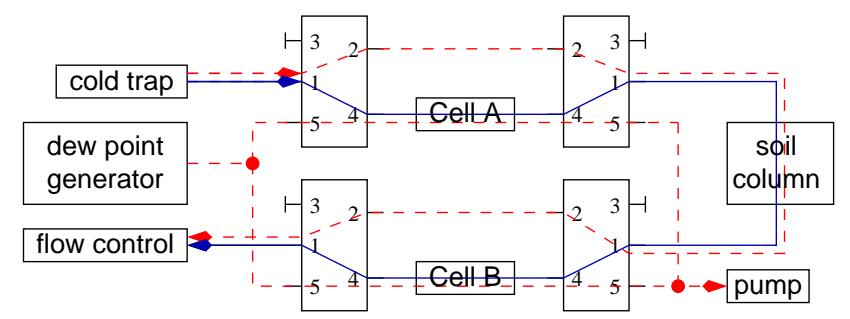

Fig. 5. Setup for runtime calibration. In normal operation (blue line), all valves are in state (1). During calibration (red dashed lines), the four valves are set to state (2), the measured gas bypasses the gas analyser and conditioned air from the dew point generator is flowing through both cells.

B. Several dew point values were set and the corresponding measured water vapour partial pressure $p_{\text {meas }}$ were recorded. Previously, all zero and span calibrations had been made. The partial pressures corresponding to the nominal dew point values were calculated using Magnus' formula, Eq. (4). As can be seen in Fig. 4, the instrument is quite linear through the measured range and the deviation from the nominal molar fraction is small.

\subsection{Runtime calibration}

Since a typical evaporation experiment can last for several weeks, the gas analyser must be calibrated during runtime. This is done by switching the measurement gas stream using bistable magnetic valves, such that it flows through a bypass. Then, calibration gas is flown through both cells of the gas analyser, and when values become stable, the calibration routines are executed. No measurements can be taken during that time. After calibration completed, it is switched back to normal operation. The setup is sketched in Fig. 5.

It is generally desirable to keep the evaporation conditions during calibration as steady as possible, to ensure that the data can validly be interpolated during that time. While calibration is running, the conductivity of the system the measurement gas goes through increases: the bypass has a higher conductivity than the measurement cells (including the air filters). To keep the flow rate through the system constant, the pump rate is lowered during calibration. Accordingly, the pressure in the evaporation chamber changes. This does not matter however, as the evaporation process is not sensitive to pressure changes. When switching back, a small amount of residual calibration gas enters the evaporation chamber. This has virtually no effect on the evaporation process, because the volume of the measurement cells is neglectable compared to the incoming air stream: $\tau=V_{\text {cell }} / q \approx 11 \mathrm{~cm}^{3} / 500 \mathrm{l} / \mathrm{h} \approx 2.3 \mathrm{~min} \ll \tau_{\text {dynamics }}$. Here, $\tau_{\text {dynamics }}$ is the internal time scale of the soil during the evaporation experiment. It is on the order of some hours as may be deduced from Fig. 11.

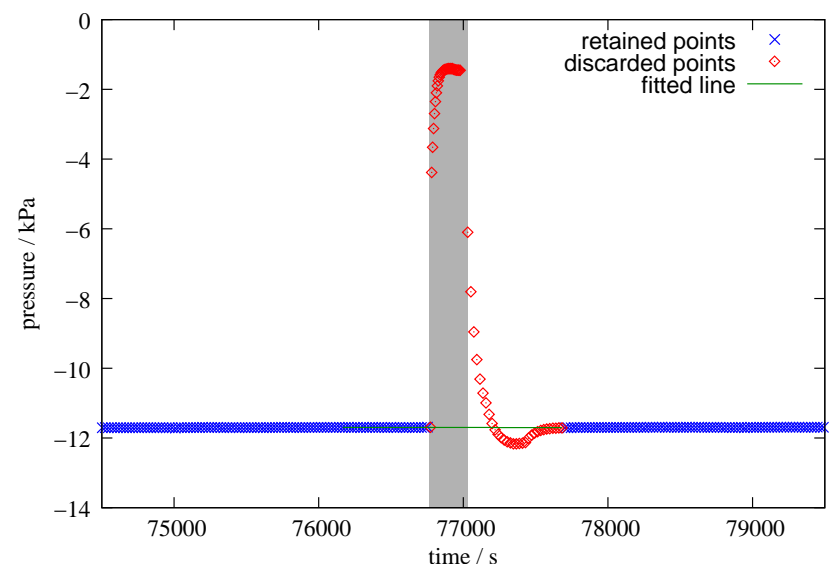

Fig. 6. Filtered data of the pressure in the evaporation chamber. The data is displayed as points, the ones which were discarded in red and the ones retained in blue. The fitted line is displayed in green, the time of the actual calibration process is marked as a gray band.

\subsection{Gas flow measurement}

The gas flow through the system is measured by the pressure drop on a capillary. With laminar flow, the relation between the gas flow $q$ and the pressure difference $\Delta p$ is linear. As the flow will not be totally laminar, a second order polynomial was used. The coefficients $a_{i}$ were determined by a calibration measurement: The flow $q$ was measured with a rotameter, several flow rates were set and the corresponding $(\Delta p, q)$ data pairs were recorded. Then the polynomial was fitted through the data.

\subsection{Data filtering}

As mentioned in Sect. 3.3, the pressure in the measurement system changes during runtime calibration. These pressure peaks need some time to decay after switching back to normal mode. As explained above, the pressure jumps do not disturb physical information. However they bother data continuity, because the gas analyser is sensitive to pressure jumps. Therefore, the pressure peaks are filtered out (Fig. 6): for each calibration process, a line is fitted through the last 20 data points before the calibration had started using linear regression. The first point after the time when the calibration process finished whose value is within twice the standard deviation of the line is defined as end point. The time difference between the end point and the end of the calibration process is limited to $10 \mathrm{~min}$. All points between the start of the calibration and the end point are discarded.

\subsection{Water flux at the upper boundary}

The water flux is calculated from the total air flow through the system $q$ and the difference of the molar fraction of water 


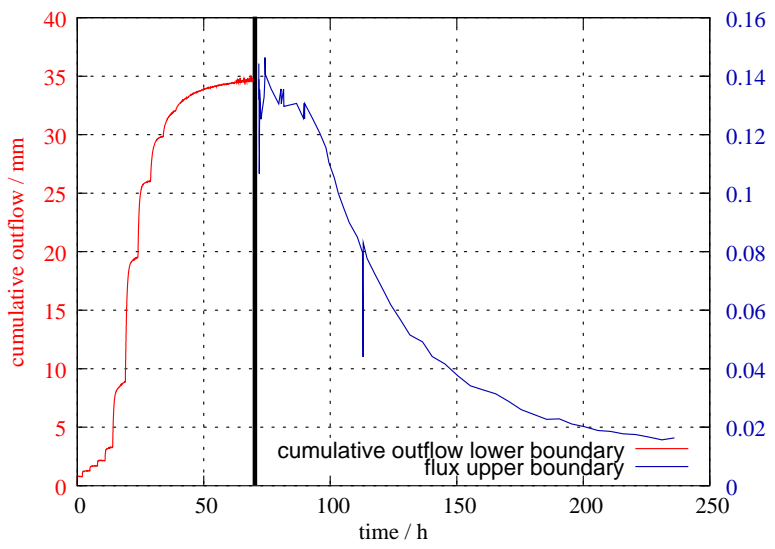

Fig. 7. Measured outflow of the sand sample. The red line shows the cumulative outflow at the lower boundary during MSO, the blue line the flux rate at the upper boundary during the evaporation experiment. The black vertical line marks the switch between MSO and evaporation.

vapour $x_{d}=x_{B}-x_{A}$. For the flow in units of mole,

$\dot{v}_{g}^{w}=x_{d} \dot{v}_{g}$

where $\dot{v}_{g}^{w}$ denotes water vapour flow and $\dot{v}_{g}$ total gas flow, both in units of mole. It is assumed that the water vapour molar fraction is constant during one measurement, because the measurement is very fast compared to the time scale of soil dynamics. Employing the ideal gas law, the final relation

$j_{\exp }^{w}=\frac{V_{m}^{w}}{R A} \frac{x_{d} p q}{T}$

for the water flux at the upper boundary $j_{\exp }^{w}$ is obtained, where $p$ is the total pressure in the evaporation chamber and $A$ the area of the soil surface.

\section{Inverse modelling}

Hydraulic parameters were estimated from the evaporation measurements on the undisturbed soil sample using inverse modelling. We used a numerical forward model together with the Levenberg-Marquardt algorithm. The forward model integrated Richards' equation using a cell-centred finite-volume scheme with full-up-winding in space and an implicit Euler scheme in time. Linearisation of the nonlinear equations is done by an inexact Newton method with line search. The linear equations are solved with a direct solver. For the time solver the time step is adapted automatically. A no-flux condition was used for the lower boundary. At the upper boundary the evaporation was calculated by Eq. (13). This is a nonlinear boundary condition using the measured quantities $T$ and $p_{\exp }^{w}$ and the matric potential at the soil surface $\psi_{m}^{b}$ and was implemented in the forward model. To account for the temperature dependence of the equivalent conductivity of the vapour phase the measured temperature at

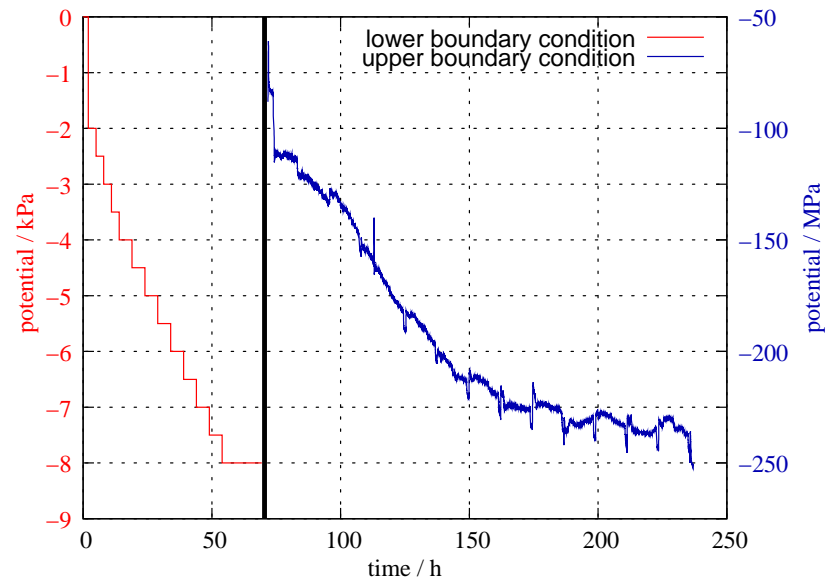

Fig. 8. Boundary conditions during the experiment with the sand sample. The red line shows the lower boundary condition during the MSO, the blue line the upper boundary condition during the evaporation experiment. A no-flow boundary condition was set at the upper boundary during MSO and at the lower boundary during evaporation. The black vertical line marks the switch between MSO and evaporation.

the upper boundary was always used for the whole soil. The sensitivities required by the Levenberg-Marquardt algorithm were derived by external numerical differentiation.

\section{Results}

Two test measurements were made, one with a sand sample and one with an undisturbed sample from a sandy loam soil.

\subsection{Sand sample}

Sand with grain size below $0.25 \mathrm{~mm}$ (density $\rho_{s}=2.65 \mathrm{~g} / \mathrm{cm}^{3}$ ) was filled in the PVC sample cylinder. The sand was filled into water, such that the water level was always above the sand, to prevent entrapped air. The evaporation chamber including the vertical TDR sensor was installed at the top of the sample.

Generally, the new method can be started at saturation. However, as the main objective of this experiment was to test the evaporation method in the dry range, an MSO experiment was run for the saturated to semi-dry range to reduce experimental runtime by setting the initial condition for the evaporation experiment. MSO experiments are particularly fast for sandy samples as the hydraulic conductivity in the wet range is very high. Note that intrinsically MSO is not needed for our method as is demonstrated in Sect. 5.2.

The lower boundary condition was first set to $0 \mathrm{kPa}$ for $2 \mathrm{~h}$ and then changed in $0.5 \mathrm{kPa}$ steps from $-2 \mathrm{kPa}$ to $-8 \mathrm{kPa}$, each step lasting $3 \mathrm{~h}(0.1 \mathrm{kPa}$ correspond to $1 \mathrm{~cm}$ water column). The last step was continued until $69.4 \mathrm{~h}$, where the MSO was terminated. The water was removed from the base 


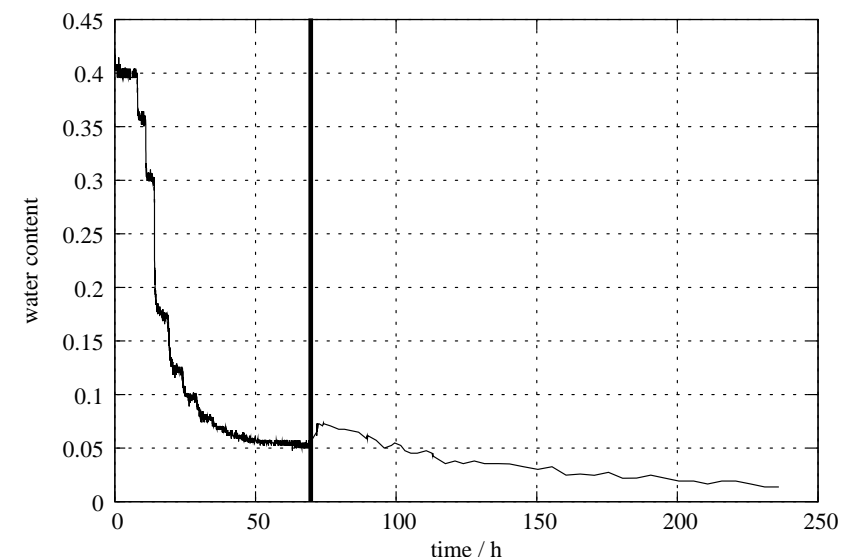

Fig. 9. Total water content of the sand sample, measured by TDR. The black vertical line marks the switch between MSO and evaporation.

at the lower boundary while the pressure was still applied to the bottom, to prevent back-flow of water into the sample. Then the evaporation experiment was started. At $t=236.9 \mathrm{~h}$, the experiment was terminated.

Figure 7 shows the outflow at the lower boundary during MSO and the flux density at the upper boundary during evaporation, respectively. The shape of the evaporation flux is as one would expect, with a rapid decay to a zero flux: The conductivity decreases rapidly as the sample dries out.

The peaks at $t=113 \mathrm{~h}$ were caused by an aberration in cold trap temperature: for $10 \mathrm{~min}$, the temperature deviated by maximal $+2.3{ }^{\circ} \mathrm{C}$ from its nominal value. This resulted in a higher $\mathrm{H}_{2} \mathrm{O}$ concentration in the incoming air stream, hence a higher potential (Fig. 8). This jump in potential caused a smaller evaporation rate, which is seen in the flux, Fig. 7. This demonstrates the high sensitivity of the experimental setup.

The total water content was measured by TDR (Fig. 9). The increase upon switching from MSO to evaporation is caused by water in the ceramic plate which is required in the MSO setup. This plate is $13 \mathrm{~mm}$ thick and has the same diameter as the soil sample. Its porosity is 0.34 with air entry value of about $-1.2 \mathrm{kPa}$. Directly above the ceramic plate is a porous membrane with an air entry value of $-23 \mathrm{kPa}$ which functions as phase separator. The plate and the membrane were not removed after MSO because this would have disturbed the soil sample, generating uncontrollable modifications. They were just disconnected from the rest of the system. Therefore the $4.4 \mathrm{~mm}$ water contained in the ceramic plate entered the soil sample during evaporation, as the platemembrane system drains after the switch because its air entry point towards the lower boundary is only $-1.2 \mathrm{kPa}$.

Total water content change is about $0.39,0.057$ of it during evaporation. This corresponds to a total outflow of $39 \mathrm{~mm}$ (5.7 $\mathrm{mm}$ during evaporation). Integrating the flux during the evaporation period results in a total cumulative out-

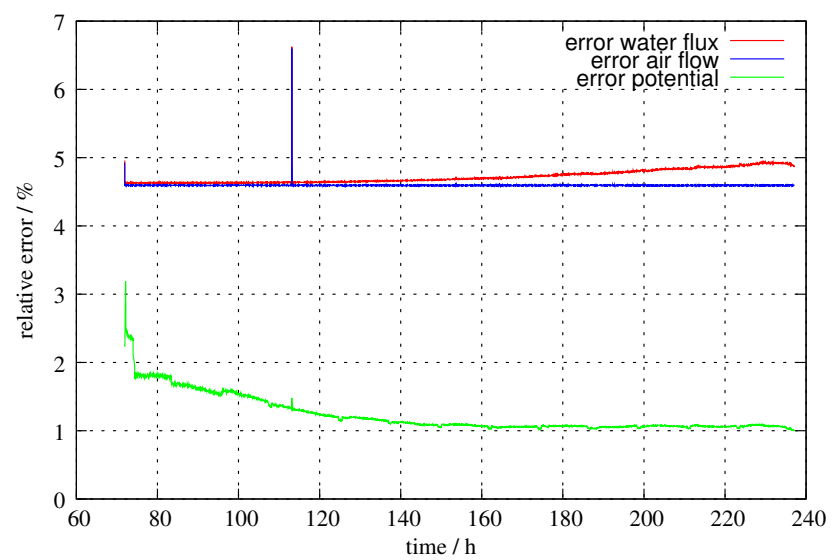

Fig. 10. Relative error of the evaporation measurements of the sand sample.

flow of $8.9 \mathrm{~mm}$. The total outflow (MSO and evaporation) is $43.8 \mathrm{~mm}$. The difference is explained quite well by the $4.4 \mathrm{~mm}$ water which entered the sample from the ceramic plate. The remaining difference is attributed to a disturbance of pore geometry by the TDR probe. When the sensor is inserted into the sand, the soil matrix is modified such that round the rods larger pores are created. Thus, the pore size distribution surrounding the TDR probe is slightly changed to larger pores. When the potential becomes more negative, these larger pores are drained first, thus the TDR sensor detects a smaller water content which is not representative for the whole sample. This effect is also demonstrated by MSO: The water content measured by TDR shows large outflow at smaller potentials than actually recorded at the lower boundary (compare Figs. 7 and 9).

Figure 10 shows the relative error of the evaporation measurements. The error of the gas analyser measurement was estimated from the test measurements (Sect. 3.2). Absolute concentration was calibrated every hour, thus obtaining a worst-case error of $0.05 \mathrm{mmol} / \mathrm{mol}$, relative concentration was calibrated every 8 hours leading to $0.016 \mathrm{mmol} / \mathrm{mol}$ worst-case uncertainty in the concentration difference. The error of the gas flow measurement was estimated using the noise of the pressure transducer and the error of the coefficients of the polynomial, which was determined by the fit program. The latter include the error of rotameter and pressure readings during calibration, as these errors were given as weights to the fit function. The accuracy of the temperature and pressure measurement was $\pm 0.2 \mathrm{~K}$ and $\pm 0.05 \mathrm{kPa}$, respectively. Because the pressure transducer in the evaporation chamber only measured relative pressure changes, the absolute pressure was determined at the start of the experiment. The error of this pressure reading was $0.5 \mathrm{kPa}$. All errors were calculated using Gaussian error propagation.

As absolute values of the error vary during the experiment, the relative error was plotted against time. The water 


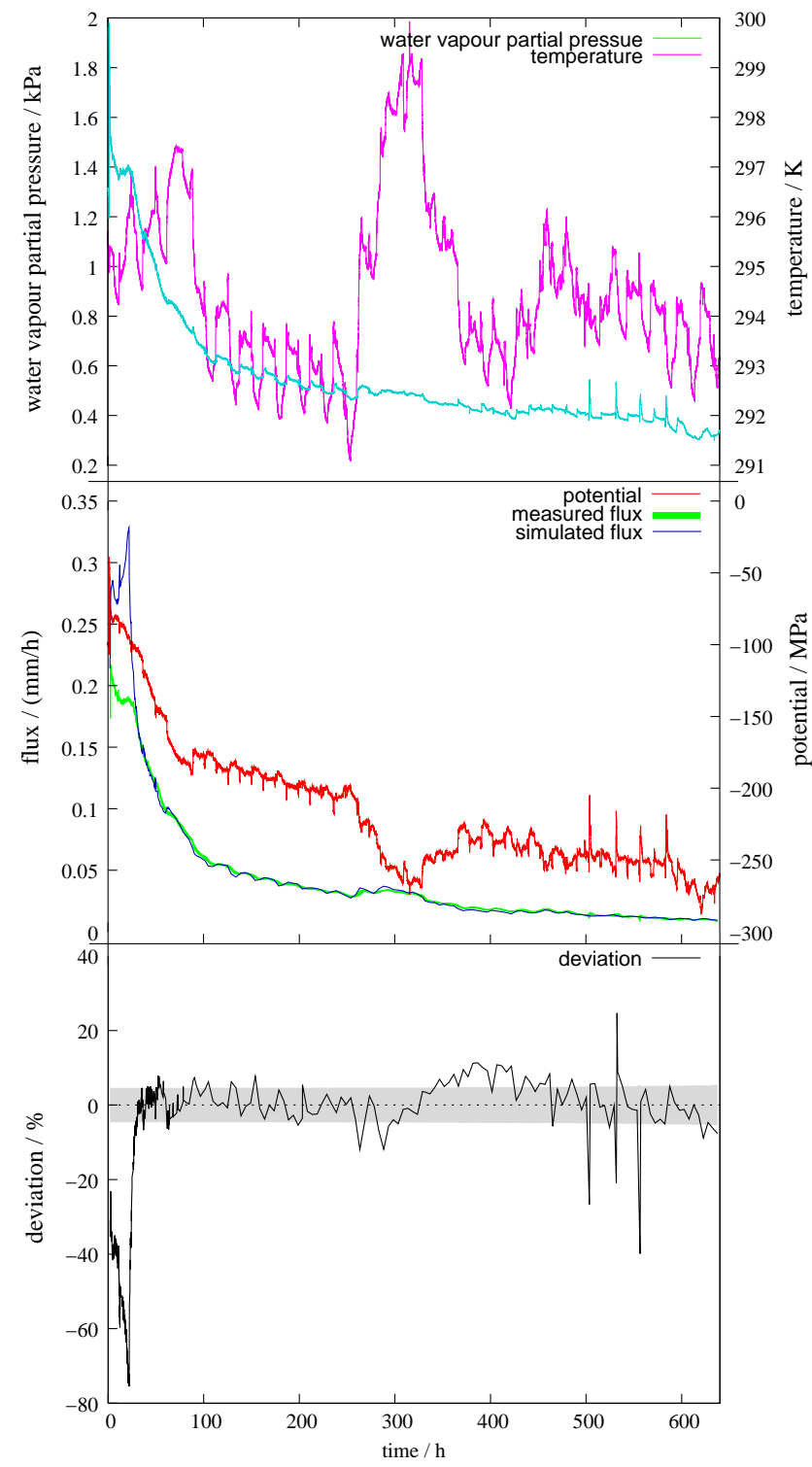

Fig. 11. Results of the evaporation experiment with the undisturbed soil sample. The thickness of the measured flux curve in the middle frame denotes the standard deviation. Notice that measured and simulated flux (middle frame) practically overlap. The relative deviation $\left(j_{\exp }^{w}-j_{\text {model }}^{w}\right) / j_{\exp }^{w}$ is shown in the bottom frame together with the measuring uncertainty from Fig. 13 (gray band).

flux error is about $4.5 \%$ to $5 \%$, the error of the boundary condition about $1 \%$ to $2 \%$. The major part of the error of the water flux results from the gas flow measurement using the capillary. Thus, the accuracy can be improved easily by using a more precise gas flow measurement device. For a gross estimation of absolute error values, typical values are $p_{\text {tot }}=(90 \pm 0.5) \mathrm{kPa}$ for the absolute pressure in the evaporation chamber, $T=(295 \pm 0.2) \mathrm{K}$, and $x=(6 \pm 0.05) \mathrm{mmol} / \mathrm{mol}$. With these values one obtains a vapour pressure precision of $\pm 5 \mathrm{~Pa}$ and a potential of $(-215 \pm 2) \mathrm{MPa}$. On the other hand, at $95 \%$ relative humidity, we would have $x=(27.71 \pm 0.05) \mathrm{mmol} / \mathrm{mol}$ and $p_{w}=(2.49 \pm 0.01) \mathrm{kPa}$, but $\psi=(-7.0 \pm 1.8) \mathrm{MPa}$ as the potential depends logarithmically on the vapour pressure. At $99 \%$ humidity, it would even be $\psi=(-1.4 \pm 1.8) \mathrm{MPa}$ which is certainly an upper limit for measurable potentials.

\subsection{Undisturbed soil sample}

Soil hydraulic properties have to be estimated for a wide range of water contents. However, for fine-grained materials, MSO experiments can only cover the very wet range. The gain of runtime from an initial MSO experiment is thus much smaller than for coarse-grained materials. As a consequence, the evaporation experiment with the undisturbed soil sample was started at saturation. This also avoids the flow reversion when switching from MSO to evaporation and thus hysteresis. The latter is generally difficult to model since it is not yet understood sufficiently well.

The sample was taken from a field site directly into the PVC sample cylinder. It was then slowly saturated from below with deionised water with $0.352 \mathrm{mmol} / 1 \mathrm{CaCl}_{2}$ until the hydraulic potential at the bottom of the sample was equal to the the static gravimetric pressure of the sample height, $\psi^{w}=\rho_{\ell}^{w} g \eta$, and no more water was flowing in. Then bottom and top of the sample were closed and the sample was allowed to equilibrate for some days. Finally the evaporation chamber was installed on top and the experiment was started. It was run for $640 \mathrm{~h}$.

Notice that while the boundary condition (Fig. 11) suggests that the experiment only covers very low potentials the maximal value of the boundary condition is $-39 \mathrm{MPa}-$ in the soil itself the whole potential range from $0 \mathrm{kPa}$ onwards is encountered as the experiment started at saturation.

The measured flux and potential is shown in Fig. 11. Integrating the flux resulted in a cumulative outflow of $(27.1 \pm 1.3) \mathrm{mm}$. Weighing the sample before and after the experiment yielded $(26.5 \pm 0.5) \mathrm{mm}$. The aberration in the boundary condition around $t=300 \mathrm{~h}$ was caused by heating of the laboratory. Its result on evaporation can be seen in the flux rate and further corroborates the correctness of the data.

Figure 12 shows the total water content, measured by TDR. The permittivity of the soil matrix was determined based on volumetric porosity and water content measurements as well as TDR bulk permittivity measurements on the field site when the sample was taken during an excavation. The total change in water content is $(25 \pm 3) \mathrm{mm}$. This is consistent with the flux and weight measurements. The figure also shows the mass balance $\eta \theta_{l}(t)+\int_{0}^{t} j_{\exp }^{w}(\tau) d \tau$, where $\eta$ denotes the height of the sample. It is assumed to be constant during the whole measurement. The decrease at the beginning is again attributed to larger pores around the TDR rods which were created by the insertion of the probe, analogous to the sand sample. Because these larger pores are drained first, the measured water content was not representative for 


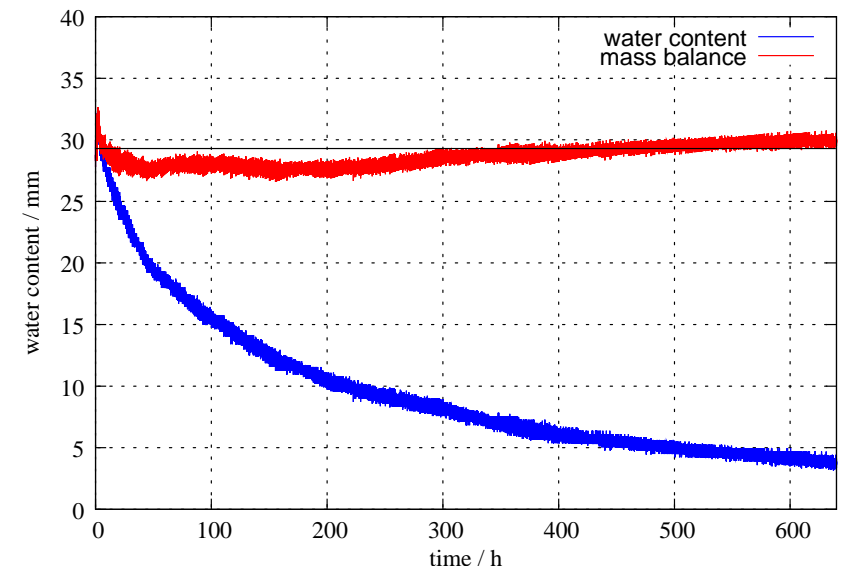

Fig. 12. Total water content (expressed as equivalent height of water in the soil column) of the undisturbed soil sample, measured by TDR (blue), and the mass balance $\eta \theta_{l}(t)+\int_{0}^{t} j_{\exp }^{w}(\tau) d \tau$ (red). The black horizontal line represents the initially measured water content.

the whole sample. As the potential became more negative, the smaller pores in the undisturbed part of the sample were drained as well and the real water content again matched the one measured by TDR. The relative error is shown in Fig. 13. In this particular experiment, the absolute pressure at startup had an error of $1 \mathrm{kPa}$. Again, the error of the potential is $1 \%$ to $2 \%$ and water flux error $4.5 \%$ to $5 \%$. This high data quality is crucial for the quality of the result of the inversion process.

Hydraulic parameters were estimated from the measured values using the inverse model described in Sect. 4. Only evaporation rates were used as target variables. Fitted parameters are the Brooks-Corey parameters $\lambda$ and $\psi_{e}$, the saturated hydraulic conductivity $K_{s}$, the available water content $\theta_{s}-\theta_{r}$, and the effective thickness $r_{b}$ of the boundary layer. The value of $\tau$ was fixed at 0.5 as suggested by Mualem (1976). The amount of data used for the inversion process was reduced by filtering to keep the model runtime reasonable. Data points were retained if the difference to the last kept point was $\Delta p_{w} \geq 15 \mathrm{~Pa}$ partial pressure or $j_{w} \geq 0.005 \mathrm{~mm} / \mathrm{h}$ water flux, or if the time step $\Delta t$ was greater than $5 \mathrm{~h}$. A grid convergence study gave a necessary spatial resolution of $0.125 \mathrm{~mm}$ equivalent to 880 grid points.

Figure 11 illustrates that for times $t>30 \mathrm{~h}$, the optimised model response is in excellent agreement with the data. For shorter times, however, the model is obviously not capable to describe the data. This may be explained by thermal processes that are not represented in the model. At the start of the experiment, the entire column is in thermal equilibrium. With the onset of evaporation, latent heat is consumed right at the saturated soil surface. As a consequence, the temperature drops there and with it the vapour pressure of water. Hence, with Eq. (13), the evaporation flux will be reduced. In its current formulation, our model does not include the effect of latent heat and therefore yields a gross over-prediction

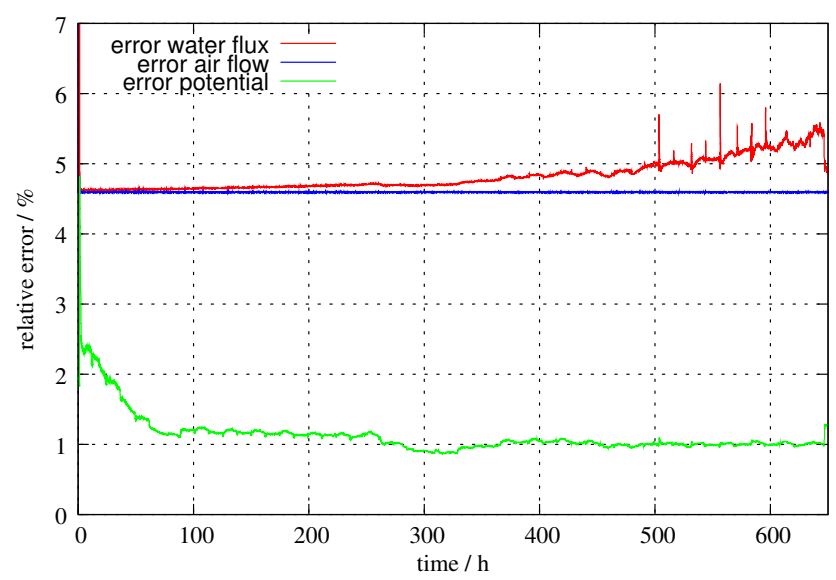

Fig. 13. Relative error $(1 \sigma)$ of the evaporation measurement of the undisturbed soil sample.

of the evaporation flux. An analytical estimation of the order of magnitude of that effect fitted well with the observed one. As a consequence, the data for $t<30 \mathrm{~h}$ were not used for the inversion.

Once the soil surface starts to dry, the effect of latent heat drops very rapidly because: (i) the evaporation flux becomes smaller, due to the decreasing hydraulic conductivity, and with it the rate of latent heat consumption, (ii) with the rapid widening of the region where evaporation occurs, heat is extracted from a larger volume such that the local temperature depression decreases, and (iii) in the initial phase the strongly temperature-dependent boundary layer limits the evaporation flux whereas later the less temperature-sensitive hydraulic properties become determining. This leads to the eventual excellent agreement between model and data. In particular we notice that the aberration caused by the heating of the laboratory is easily reproduced by the model. While such an agreement is no prove that the model is correct, it is a strong hint that it may be used as an effective representation of the real system under similar conditions as those encountered during the experiment. The resulting parameters and their standard deviations resulting from the analysis of the sensitivity matrix are given in Table 1 . While we do not have any independent confirmation, they appear reasonable for the soil under examination.

\section{Conclusions}

We presented a novel experimental approach to evaporation experiments for determining soil hydraulic properties. A detailed error analysis demonstrated the very high accuracy of the new technique which is further confirmed by the rapid and consistent response of the measured flux to small fluctuations of the boundary condition.

The high data quality and the correct representation of the underlying physics are crucial for the quality of the 
Table 1. Resulting parameters and their standard deviations resulting from the analysis of the sensitivity matrix for the undisturbed soil sample.

\begin{tabular}{llll}
\hline parameter & fitted value & \multicolumn{2}{c}{ standard deviation } \\
\hline$\lambda(-)$ & 1.03 & \pm 0.1 & $(10 \%)$ \\
$\psi_{e}(\mathrm{~Pa})$ & 1020 & \pm 40 & $(4 \%)$ \\
$K_{S}(\mathrm{~cm} / \mathrm{h})$ & 0.116 & \pm 0.009 & $(8 \%)$ \\
$\theta_{S}-\theta_{r}\left(\mathrm{~m}^{3} / \mathrm{m}^{3}\right)$ & 0.306 & \pm 0.002 & $(1 \%)$ \\
$r_{b}(\mathrm{~mm})$ & 2.74 & \pm 0.05 & $(2 \%)$ \\
\hline
\end{tabular}

numerical inversion. The excellent agreement between measured and simulated data for longer times are a strong indication that all the relevant processes, within our window of view, are captured by Richards' equation with an effective hydraulic conductivity function that explicitly incorporates vapour transport and with a constant effective diffusive boundary layer at the soil-atmosphere interface. For shorter times, the discrepancy between data and model require the inclusion of additional processes. Qualitatively, the deviations can be understood in terms of latent heat consumed by the evaporating water. In the current model formulation, this process and the associated transport of heat is not included.

In this publication we focussed on technical issues of the new method. A detailed analysis of the inversion method to further investigate the parameter estimation procedure is needed for a full evaluation of the quality of the inverted parameters. This will be investigated in a later study.

The main advantage of our new approach over traditional evaporation experiments is that it yields data right from the soil surface to which the inversion is most sensitive. Practical advantages include (i) a large and uniform measurement range that is achieved by directly measuring the flux and by the boundary condition which can be controlled in a wide range through the air flow and air conditioning and (ii) a constant and rather high accuracy for arbitrarily sized soil samples. In addition, the method is applicable directly in the field after minor modifications and it is an excellent tool for detailed studies of evaporation from porous media. With all the advantages, a final caveat is in order. The cost of the instrumentation is rather high when commercial equipment is used.

\section{Appendix A}

\section{List of symbols}

Superscripts $w$ and $a$ denote water and air, respectively. Subscripts $g$ and $\ell$ denote the gas phase and the liquid phase, respectively.

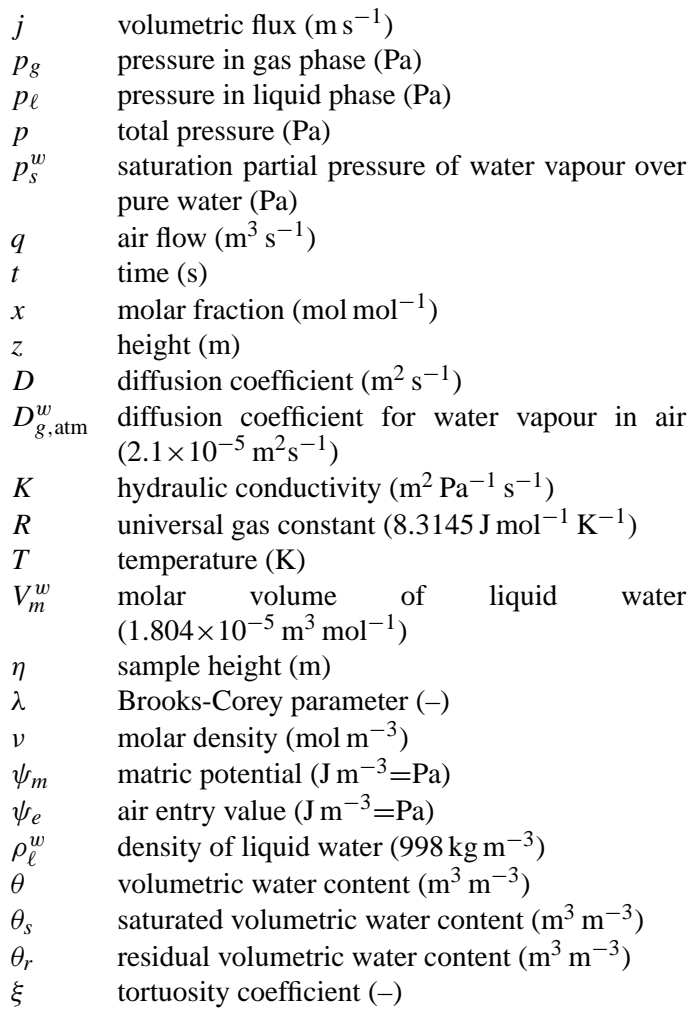

Acknowledgements. We acknowledge and greatly appreciate the constructive comments of two anonymous reviewers and of N. Romano.

Edited by: N. Romano

\section{References}

Eching, S. O., Hopmans, J. W., and Wendroth, O.: Unsaturated hydraulic conductivity from transient multistep outflow and soil water pressure data, Soil Sci. Soc. Am. J., 58, 687-695, 1994.

Gardner, W. R. and Miklich, F. J.: Unsaturated conductivity and diffusity measurements by a constant flux method, Soil Sci. Soc. Am. J., 93, 271-274, 1962.

Ippisch, O., Vogel, H.-J., and Bastian, P.: Validity limits for the van Genuchten-Mualem model and implications for parameter estimation and numerical simulation, Adv. Water Resour., 29, 17801789, 2006.

Jin, Y. and Jury, W. A.: Characterizing the dependence of gas diffusion coefficient on soil properties, Soil Sci. Soc. Am. J., 60, 66-71, 1996.

Jury, W. A., Gardner, W. R., and Gardner, W. H.: Soil Physics, John Wiley \& Sons, New York, 5th edn., 1991.

Mualem, Y.: A new model for predicting the hydraulic conductivity of unsaturated porous media, Water Resour. Res., 12, 513-522, 1976.

Murray, F. W.: On the computation of saturation vapor pressure, J. Appl. Meteorol., 6, 203-204, 1967.

Parker, J. C., Kool, J. B., and van Genuchten, M. T.: Determinig soil hydraulic properties from one-step outflow experiments by pa- 
rameter estimation: II. Experimental studies, Soil Sci. Soc. Am. J., 49, 1354-1359, 1985.

Philip, J. R. and de Vries, D. A.: Moisture movement in porous materials under temperature gradients, Trans. Am. Geophys. Union (EOS), 38, 222-232, 1957.

Rawlins, S. L. and Campbell, G. S.: Water potential: Thermocouple psychrometry, in: Methods of soil analysis, Part 1. Physical and mineralogical methods, edited by: Klute, A., Agronomy Series 9, 597-618, Am. Soc. Agron., Madison, WI, 2nd edn., 1986.

Robinson, D. A., Jones, S. B., Wraith, J. M., Or, D., and Friedman, S. P.: A review of advances in dielectric and electrical conductivity measurement in soils using time domain reflectometry, Vadose Zone J., 2, 444-475, 2003.

Romano, N. and Santini, A.: Determining soil hydraulic functions from evaporation experiments by a parameter estimation approach: Experimental verifications and numerical studies, Water Resour. Res., 35, 3343-3359, 1999.
Šimůnek, J., Wendroth, O., and van Genuchten, M. T.: Parameter estimation analysis of the evaporation method for determining soil hydraulic properties, Soil Sci. Soc. Am. J., 62, 894-905, 1998.

Tamari, S., Bruckler, L., Halbertsma, J., and Chadoeuf, J.: A simple method for determining soil hydraulic properties in the laboratory, Soil Sci. Soc. Am. J., 57, 642-651, 1993.

Topp, G. C. and Miller, E. E.: Hysteretic moisture characteristics and hydraulic conductivities for glass-bead media, Soil Sci. Soc. Am. Proc., 30, 156-162, 1966.

van Dam, J. C., Stricker, J. N. M., and Droogers, P.: Inverse method to determine soil hydraulic functions from multistep outflow experiments, Soil Sci. Soc. Am. J., 58, 647-652, 1994.

Wendroth, O., Ehlers, W., Hopmans, J. W., Kage, H., Halbertsma, J., and Wösten, J. H. M.: Reevaluation of the evaporation method for determining hydraulic functions in unsaturated soils, Soil Sci. Soc. Am. J., 57, 1436-1443, 1993. 